\title{
MAPPING THE SURFACE DISTRIBUTION OF ELEMENTS ON AP STARS USING THE MAXIMUM ENTROPY METHOD
}

\author{
ARTIE P. HATZES \\ McDonald Observatory, The University of Texas at Austin, Austin, TX, \\ 78712
}

ABSTRACT A technique for deriving the distribution of elements on the surface of Ap stars using maximum entropy reconstruction principles is described. The technique is applied to deriving the silicon distribution on 56 Ari, CU Vir, 11 Ori and the chromium distribution on $\gamma^{2}$ Ari. Silicon on these stars is depleted at the magnetic poles and is enhanced in regions between the magnetic equator and poles. The chromium distribution on $\gamma^{2}$ Ari is markedly different than the chromium distribution seen on other Ap stars. It shows depletions at one of the magnetic poles (as do other Ap stars) but it does not show the depleted band at the equator as has been seen on $\theta$ Aur, $45 \mathrm{Her}$, and $\omega$ Her. The silicon distribution on 11 Ori also differs from that found on other stars in that it shows evidence for a depleted band, similar to what has been seen in the chromium distribution is some stars. Characteristic features in the abundance maps such as spots or bands appear to mark the location of the magnetic poles or equator so that these maps can be used to infer the magnetic field geometries on these stars. Dipole decentering parameters derived from the abundance maps yield decentering parameters of about 0.2 stellar radii. The amount of decentering seems to be correlated with rotation period (longer period Ap stars have less decentering). Horizontal diffusion can complicate the use of abundance maps to determine the field geometry. The effects of horizontal diffusion can only be understood by a proper theoretical study of its effects or by mapping the elemental distribution on Ap stars of known age.

\section{INTRODUCTION}

The spectral variations of the magnetic Ap stars are believed to be due to the inhomogeneous distribution of elements across the stellar surface. Accurate abundance maps of the surface distributions of elements on these stars is important in that they provide critical tests of theories proposing explanations for this distribution. The surface abundance pattern seem to be intimately related to the presence of strong magnetic fields in these stars, so the abundance maps may provide useful information on the magnetic field geometry. Potentially these maps can be used to derive field geometries on those stars where magnetic field measurements are difficult to obtain. 
The development of techniques for mapping the surface distribution of elements on stars has a long and rich history dating to the early attempts by Deutsch $(1958,1970)$ who solved for the surface abundance distribution by using a series expansion of the integrated quantities of the observed stellar lines such as equivalent widths and radial velocities. In recent years tremendous progress has been made in actually solving the inverse problem, namely deriving the surface distribution of elements using the information inherent in the line shapes. Pioneering work in this area was made by Goncharsky et al. (1982) who set up an integral equation relating the the observed spectral line profiles to the local equivalent widths across the stellar surface. This integral equation was solved using Tikhonov regularisation.

In this contribution I shall outline a slightly different approach to solving the inverse problem using the principles of maximum entropy reconstruction. The mathematical formalism is outlined and compared to the Tikhonov technique. The technique is then applied to deriving the surface distribution of silicon on 56 Ari, CU Vir, and 11 Ori as well as the chromium distribution on $\gamma^{2}$ Ari.

\section{MATHEMATICAL FORMALISM}

The inverse problem can be stated concisely as a matrix equation. If $\mathbf{D}$ represents a vector of the observed spectral line profiles as a function of phase and I represents an image vector of the local equivalent widths of a spectral line across the star which we seek to derive, then

$$
\mathbf{D}=\mathbf{I} \cdot \mathbf{R}
$$

where $\mathbf{R}$ represents a transfer matrix taking one from image to data space. The matrix, $\mathbf{R}$, consists of the specific intensity profiles of each image pixel as a function of rotation phase. The matrix elements are generated using model atmospheres and all information about the stellar parameters such as rotational velocity, inclination, limb darkening, etc. are contained in the matrix elements. A more detailed description of the construction of the transfer matrix elements can be found in Vogt et al. (1987) and Hatzes et al. (1989).

The solution of this matrix equation should be straightforward:

$$
\mathbf{I}=\mathbf{D} \cdot \mathbf{R}^{-1}
$$

However, many image pixels on the star are mapped into the same location in the data vector making this is a projection problem. This implies that many image solutions could satisfy Equation 1 for a given data vector. Another way of stating this is to say that the matrix $\mathbf{R}$ is noninvertible (too many variables for the number of equations) or the problem is ill-posed. Some of this nonuniqueness or ill-posedness is removed if the star has a large rotational velocity. In this case only those pixels lying along a constant radial velocity chord are mapped into the same location in the data vector. For stars whose spectral lines shapes are dominated by rotation, each observed spectral line profile represents a 1-dimensional projection of the 2-dimensional surface. By 
obtaining a time sequence of these projections as the star rotates, one can derive the 2-dimensional surface of the star. This is the essence of the Doppler imaging technique. For a star to be an 'ideal' Doppler imaging candidate it must have $v \sin i>30 \mathrm{~km} \mathrm{~s}^{-1}$ and an inclination $30<i<90^{\circ}$. However, useful Doppler images can be obtained for stars rotating as slowly as $15 \mathrm{~km} \mathrm{~s}^{-1}$.

Because many image vectors can result in the same observed data vector, a criterion is needed for choosing a solution. If one applies Occam's razor and assumes that nature prefers simplicity, then the simplest image is the 'correct' one. The entropy provides a convenient means of quantizing this simplicity. The entropy of an image is defined as

$$
S=-\sum_{j=1}^{n} p_{j} \log p_{j}
$$

where $p_{j}$ is the normalized image quantity (local equivalent width of the $j t h$ pixel normalized to the sum of all pixels). The entropy also represents the negative of the information content in an image so that the image with the maximum entropy represents one with the least amount of information and thus the 'smoothest' or simplest image. More complicated images may exist and satisfy the data, but these cannot be uniquely determined from the data set.

Any image having maximal entropy must also be able to fit the observed data and this imposes a constraint on the image. If $d_{i}$ is the observed data and $g_{i}$ represents the fit from the image solution, then a convenient measure of the misfit is provided by the $\chi^{2}$ criterion:

$$
\chi^{2}=\sum_{i=1}^{m}\left(d_{i}-g_{i}\right)^{2} / \sigma_{i}^{2}
$$

where $\sigma_{i}$ represents the error of each data point. The value of the $\chi^{2}$ from the final solution should be at some level, $\chi_{0}^{2}$, typically set by the level of the noise in the data. Clearly one should fit the data to the level of the noise and no more.

The problem of maximizing a function subject to a constraint can be solved using Lagrange multipliers. A new functional, $Q$, is maximized where

$$
Q=S-\lambda \chi^{2}
$$

and $\lambda$ is the Lagrange multiplier to be determined.

It is interesting to compare Equation 5 with that used in the Tikhonov method for solving the inversion problem. That technique seeks to minimize the discrepancy function giving the misfit between data and fit (analogous to $\chi^{2}$ ). The constraint in this case is imposed by the choice of smoothing function. That technique seeks to minimize a functional of the form

$$
Q=\lambda S+\chi^{2}
$$

where in this case $\chi^{2}$ represents the discrepancy function and $S$ is the smoothing function (not necessarily the entropy). Note the location of the Lagrange multiplier in this case. Summarizing the differences, MEM seeks to maximize the 


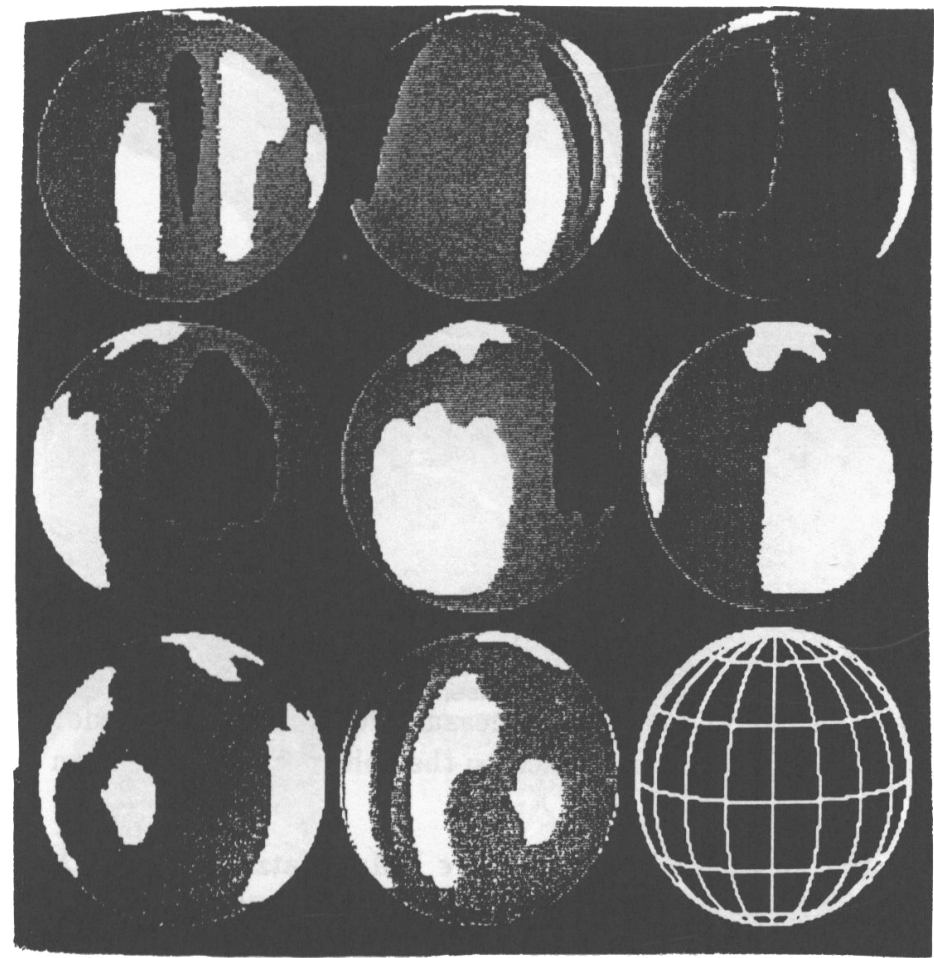

Figure 1: Abundance (local equivalent width) map of Si II $6347 \AA$ on 56 Ari. Maximum $W_{\lambda}=380 \mathrm{~m} \AA$ (white), minimum $W_{\lambda}=140$ (black), and mean $W_{\lambda}=280 \mathrm{~m} \AA$ (grey).

amount of smoothing in an image so long as that image still fits the data. The Tikhonov technique seeks to minimize the differences between the data and the fit subject to a constraint imposed by the smoothing function. Mathematically these should be equivalent. There may be subtle differences between the techniques when used on low signal-to-noise $(\mathrm{S} / \mathrm{N})$ data, but in the case of high $\mathrm{S} / \mathrm{N}$ data with adequate phase coverage and resolution both methods should produce nearly the same image.

In deriving the abundance distributions on Ap stars, the MEMSYS maximum entropy code of Gull and Skilling had been employed. Tests of the method can be found in Vogt et al, (1987) and Hatzes et al. (1989).

\section{THE DOPPLER IMAGES}

The maximum entropy method has been used to derive the distribution of chromium and silicon on a sample of magnetic Ap stars using the Si II 6347 $\AA$ and the Cr II $4824 \AA$ lines. Previous work has presented Doppler images of the silicon distribution on $\gamma^{2}$ (Hatzes, Penrod, and Vogt 1989), BP Boo (Hatzes 1990), and $\theta$ Aur (Hatzes 1991a). Chromium images have been presented for 


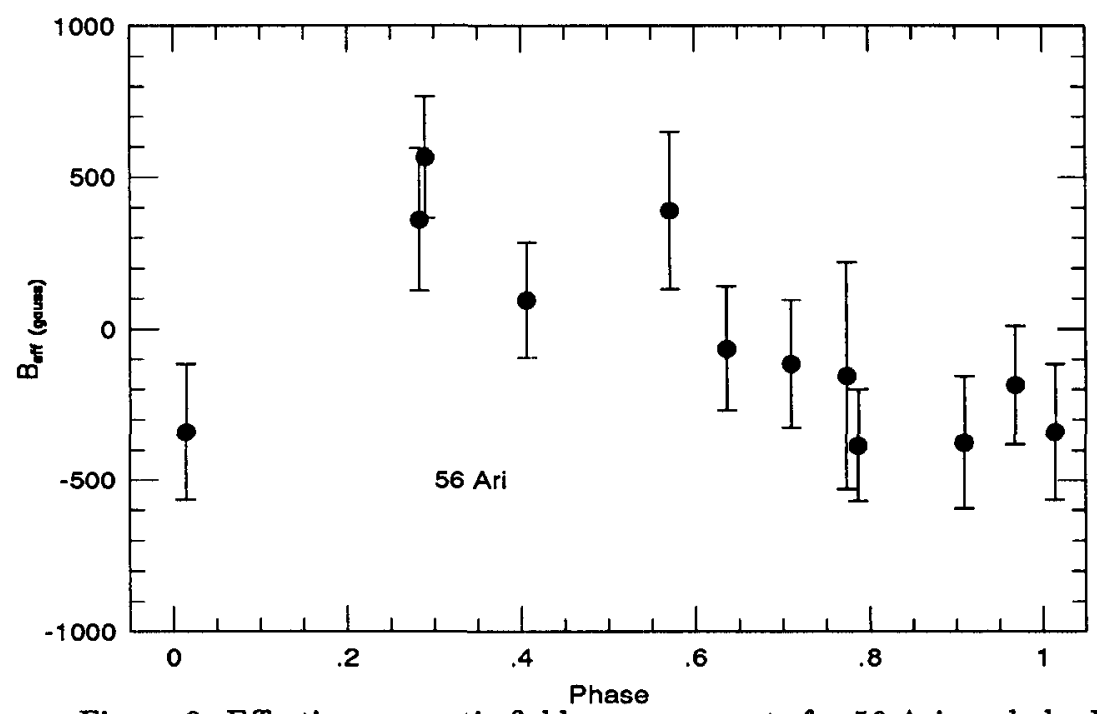

Figure 2: Effective magnetic field measurements for 56 Ari made by Borra and Landstreet (1980) and phased to the ephemeris of the silicon image.

$\theta$ Aur (Hatzes 1991a), 45 Her, $\omega$ Her, and $\epsilon$ UMa (Hatzes 1991b). Due to the lack of space only a few Doppler images that have not appeared elsewhere in the literature will be presented. In this contribution Doppler images of the silicon distribution for $56 \mathrm{Ari}, \mathrm{CU}$ Vir, 11 Ori and the chromium distribution on $\gamma^{2}$ Ari are presented.

Data were obtained using the Lick Observatory 3-m Shane telescope at the coude focus and a Texas Instruments $800 \times 8003$-phase CCD detector. The resolving power of the spectrograph setup was about 50,000 and the typical signal-to-noise of the data was about 300-500 per pixel. The data were acquired during 1985-1987.

\section{The Silicon Images}

\section{$\underline{56 \mathrm{Ari}}$}

The B9 star 56 Ari is a silicon and helium variable with a period of 0.728 days Deutsch (1955). The silicon line was observed at 19 well-spaced phases. A $\mathrm{v} \sin i$ of $160 \pm 2 \mathrm{~km} \mathrm{~s}^{-1}$ was measured for this star. The rotation period and the main sequence radius of a $\mathrm{B} 9$ star yields an inclination of $70^{\circ}$ for this star. Fig. 1 shows the resulting equivalent width map for the Si II $6347 \AA$ line at 8 equally spaced rotation phases (starting at phase 0 ) computed according to the ephemeris $2,446,662.9152+0.727895 E$. In the image black represents regions of underabundance with respect to the mean value across the star and white represents overabundance. This image represents a high contrast version of the maximum entropy image so the sharpness of features is an artifact of the display process. In reality there is a smooth variation between the abundance regions 


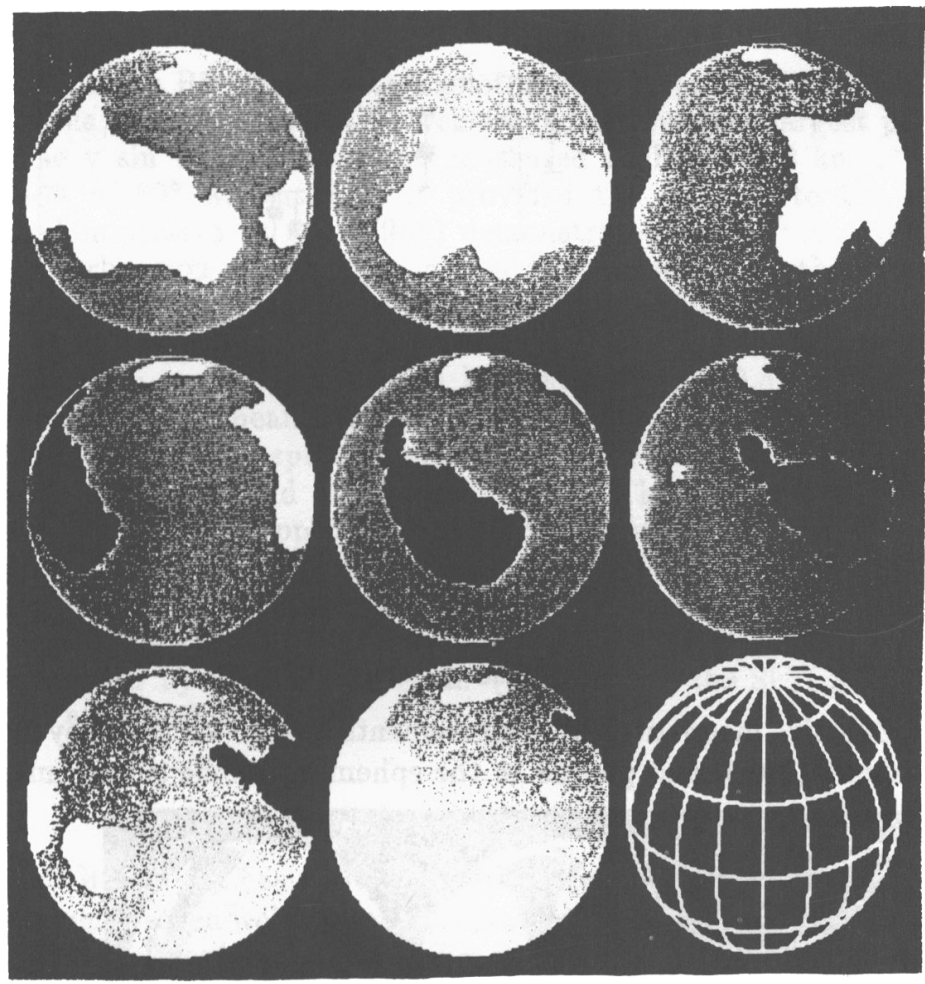

Figure 3: The silicon distribution on on CU Vir. Maximum $W_{\lambda}=600 \mathrm{~m} \AA$ (white), minimum $W_{\lambda}=180$ (black), and mean $W_{\lambda}=340 \mathrm{~m} \AA$ (grey).

on the star.

The silicon image for 56 Ari is characterized by 2 spots of depleted abundance with equivalent width, $W_{\lambda},=140 \mathrm{~m} \AA$. The smaller spot is at phase 0.0 and latitude $30^{\circ}$. The larger depleted spot is positioned near the equator at phase 0.35. The elongated shape, in latitude, of these spot features may be primarily due to the high inclination of the star. There is also a large enhanced spot $\left(W_{\lambda}=380 \mathrm{~m} \AA\right)$ centered at the equator and phase 0.55 as well as 2 smaller spots on either side of the small depleted spot. Borra and Landstreet (1980) found magnetic field variations of \pm 500 gauss for this star. These magnetic field measurements, phased to the same ephemeris as the silicon image, are shown in Fig. 2 The phase of the positive magnetic pole is coincident with the location in phase of the large depleted spot. Depleted silicon spots have also been seen at the magnetic poles of $\gamma^{2}$ Ari and $\theta$ Aur, so it seems that these features mark the location of the magnetic poles in Ap stars. If this is the case then the fact that the 2 spots are separated by less than 0.5 in phase is an indication that a large degree of dipole decentering may be present in this star. Borra and Landstreet also noted that the maximum equivalent width of the Si II $4128 \AA$ line occurred approximately 0.2 in phase after the passage of the positive magnetic pole. This is consistent with the location of the large enhanced spot shown in Fig. 1. 


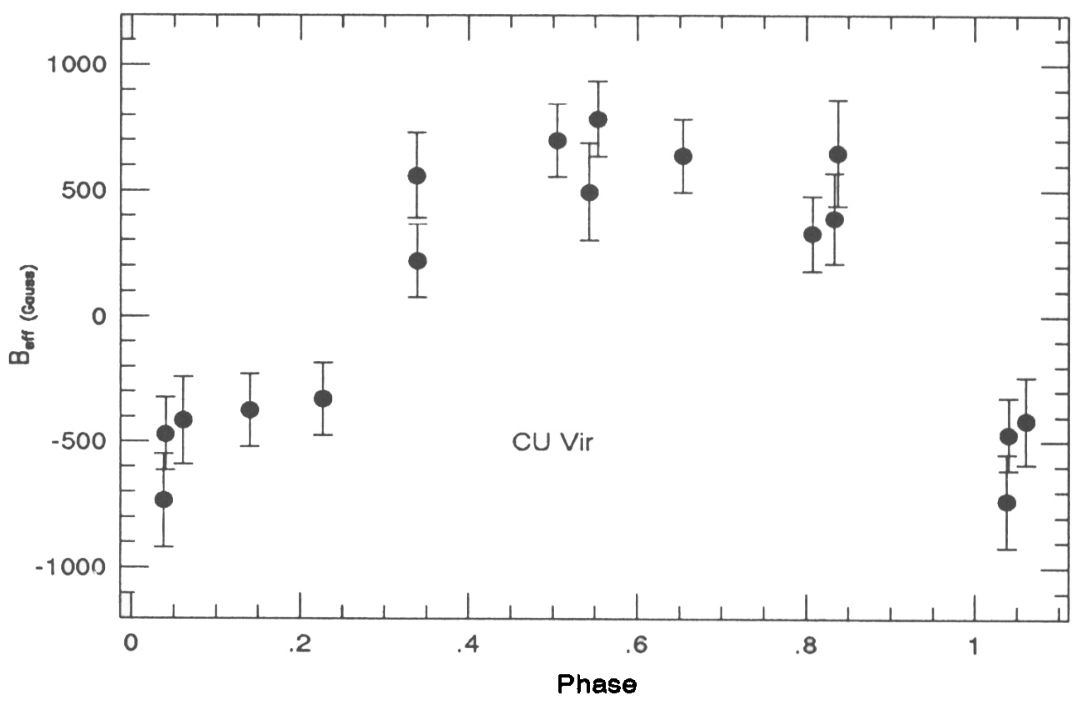

Figure 4: Effective magnetic field measurements for CU Vir made by Borra and Landstreet (1980) and phased to the ephemeris of the silicon image.

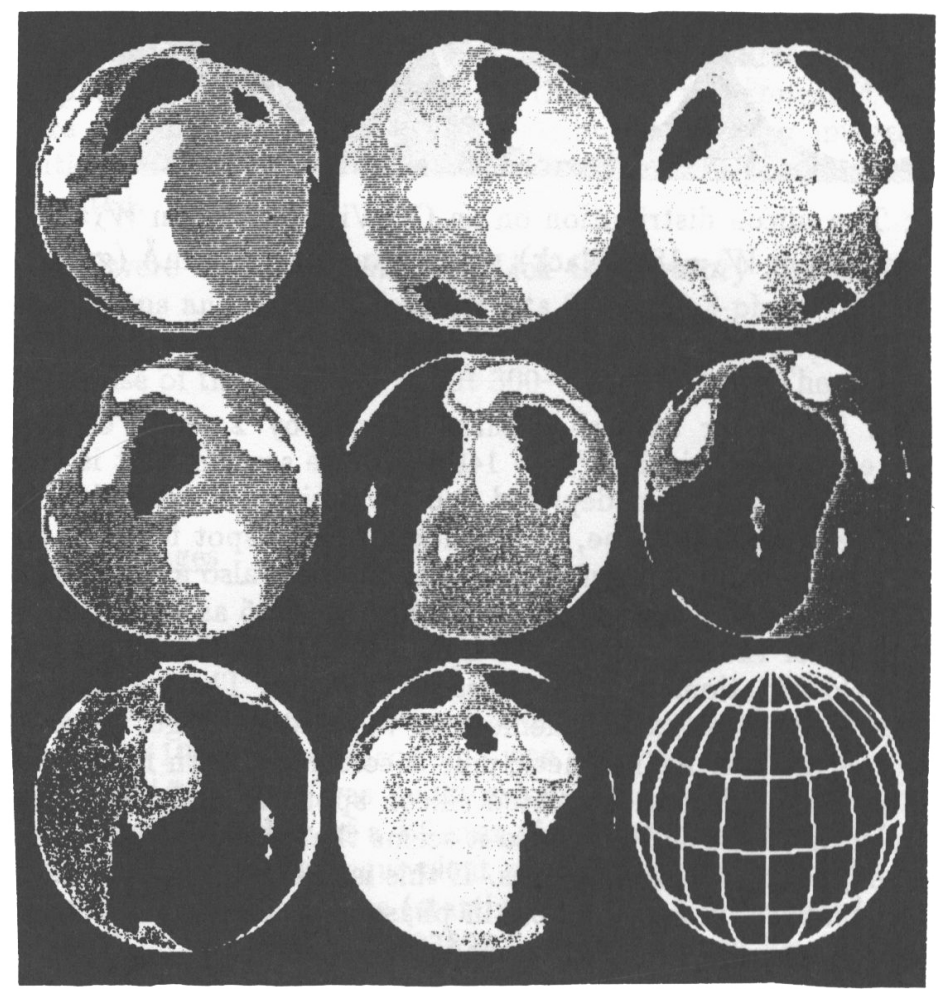

Figure 5: The silicon distribution on 11 Ori. Maximum $W_{\lambda}=320 \mathrm{~m} \AA$ (white), minimum $W_{\lambda}=65$ (black), and mean $W_{\lambda}=190 \mathrm{~m} \AA$ (grey). 


\section{$\underline{\text { CU Vir }}$}

CU Vir is a B8pSi star with a photometric period of 0.520675 days. Silicon observations were obtained at 13 well-spaced phases (the largest phase gap was 0.1 . The $\mathrm{v} \sin i$ of the star was measured to be $147 \pm 2 \mathrm{~km} \mathrm{~s}^{-1}$. A stellar inclination of $60^{\circ}$ was used as it provided the best fit to the data. Tests performed in Hatzes et al. (1989) demonstrated that $\chi^{2}$ is minimized when the true inclination of the star is used for constructing the transfer matrix elements. Fig. 3 shows the silicon distribution for this star phased to the ephemeris $2,441,455.696+0.520675 E$. It is characterized by a spot of depleted abundance $\left(W_{\lambda}=180-200 \mathrm{~m} \AA\right)$ located at phase 0.52 and latitude $+20^{\circ}$. By comparison, the mean local equivalent width across the surface of the star is about $340 \mathrm{~m} \AA$. This spot feature is very near the positive magnetic pole as determined by Borra and Landstreet (1980). These measurements, phased to the ephemeris of the Doppler image, are shown in Fig. 4. There is also a large, elongated enhanced spot $\left(W_{\lambda}=500-600 \mathrm{~m} \AA\right)$ covering an area $100^{\circ} \times 40^{\circ}$ and centered at phase 0.1 and latitude $25^{\circ}$. The center of this spot appears to be near the negative magnetic pole. This silicon distribution is consistent with the one derived by Goncharsky et al. (1983) using the Si II $3862.6 \AA$ line and Tikhonov's method.

\section{$\underline{11 \text { Ori }}$}

This B9pSi star has a rotation period of 4.6398 days (Renson and Manfroid 1981). Twenty-seven well-spaced observations were made of the Si II $6347 \AA$ line. The $\mathrm{v} \sin i$ was measured to be $50 \mathrm{~km} \mathrm{~s}^{-1}$. This along with the rotation period yields a stellar radius $>4.6 R_{\odot}$ which is much larger than the $2.8 R_{\odot}$ expected for a main sequence B9 star. The inclination of the star was taken to be $70^{\circ}$, the value that provided the lowest final $\chi^{2}$. The ephemeris for the data was taken as $\mathrm{JD}=2,446,280.9955+4.6398 E$.

The silicon distribution for this star is shown in Fig. 5. This abundance distribution looks markedly different than silicon maps seen on other stars. There is a large depleted spot $\left(W_{\lambda}=65 \mathrm{~m} \AA\right)$ of radius $15^{\circ}$ located at latitude $+40^{\circ}$ and phase 0.4 which may be the location of a magnetic pole. This spot appears to be surrounded by a partial ring of enhanced abundance $\left(W_{\lambda}=320\right.$ $m \AA$ ). Approximately 0.25 in phase after the passage of this depleted spot lies a large depleted band covering much of the visible disk. It appears that this band extends up over the equator and to the opposite side of the star. Possibly this feature represents the magnetic equator. It is not know to what extent latitudinal smearing is present in the image due to the high inclination of the star. It is possible that the apparent band is due to 2-3 individual spots that have been smeared in latitude so that they appear as a band. (In which case the silicon image would look similar to the chromium image of $\gamma^{2}$ Ari.)

\section{The Chromium Distribution on $\gamma^{2}$ Ari}

The B9p star $\gamma^{2}$ Ari is a silicon and chromium variable with a period of 1.6903 days. The effective magnetic field strength varies between -1 and +0.5 kilogauss with the same period as the spectral variations (Borra and Landstreet 1980). The measured $\mathrm{v}$ sin $i$ for this star was $64 \mathrm{~km} \mathrm{~s}^{-1}$ and the inclination of 


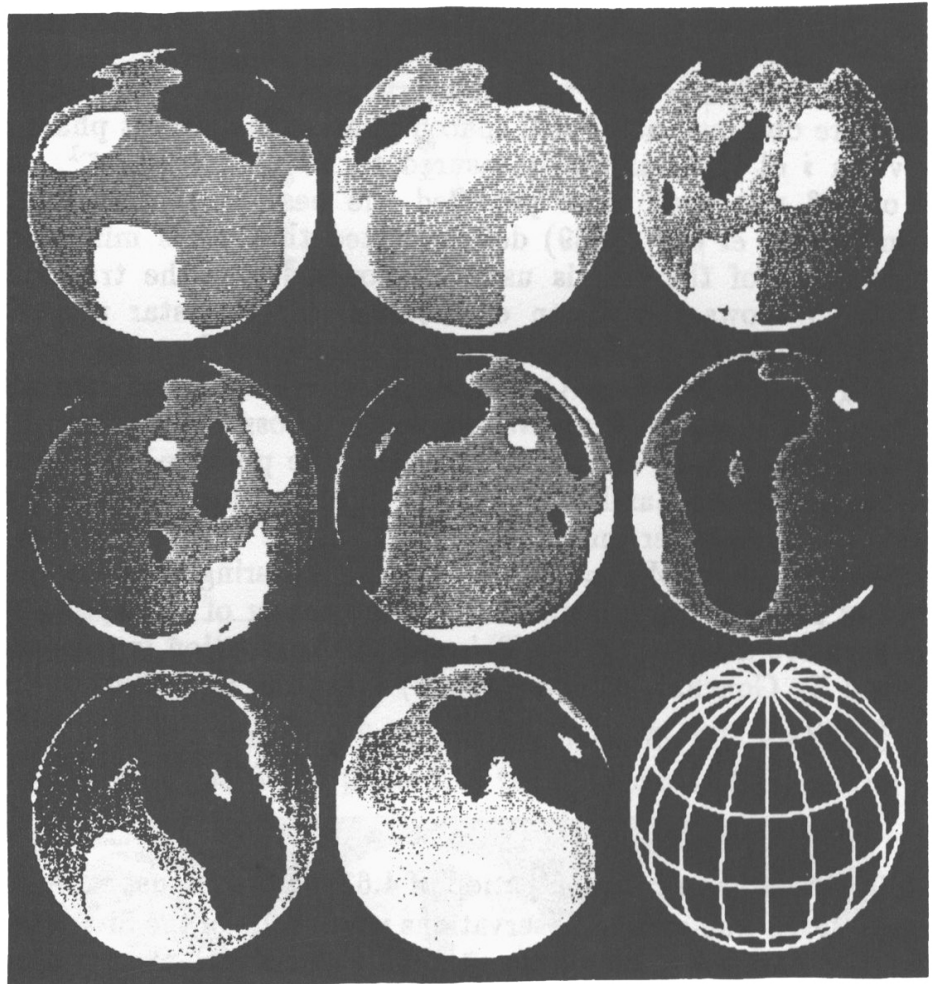

Figure 6: The chromium distribution on $\gamma^{2}$ Ari. Maximum $W_{\lambda}=200 \mathrm{~m} \AA$ (white), minimum $W_{\lambda}=140$ (black), and mean $W_{\lambda}=165 \mathrm{~m} \AA$ (grey).

$55^{\circ}$ was used in the modeling. Observations of the chromium line were made at 16 rotation phases and the resulting Doppler image is shown in Fig 6. The phasing of this image is the same as the silicon image presented in Hatzes et al. (1989). Once again depleted regions with respect to the mean value across the star are shown as black while the enhanced regions are shown as white.

The chromium image for $\gamma^{2}$ Ari shows 3 overabundant spots with equivalent widths of $180-200 \mathrm{~m} \AA$ (the mean value across the star is about $165 \mathrm{~m} \AA$ ) lying on a circle of radius $45^{\circ}$ and centered near the equator at phase 0.15 . This point is near the location of a depleted silicon spot (Hatzes, Penrod, and Vogt 1988) as well as the location (in longitude) of the positive magnetic pole (Borra and Landstreet 1980). On the opposite side of the star are 2 depleted spots (which in this reproduction appear as an elongated spot with a gap in it) with a local equivalent width of $140 \mathrm{~m} \AA$ which is only slightly below the mean value across the star. These two spots straddle a location on the star coincident with another strong depleted silicon spot located at the negative magnetic pole of the star (the location of this silicon spot is near the gap in the depleted feature of the chromium image). 
The silicon distribution of 56 Ari as well as those derived previously for other stars indicate that silicon is depleted at the magnetic poles. These spots can therefore be used to trace the location of the magnetic fields. The most promising explanation for the nonuniform distribution of elements is the selective diffusion of elements in the atmosphere of these stars first proposed by Michaud (1970). The presence of magnetic fields modifies this distribution by inhibiting the motion of ionized species across magnetic field lines. This theory predicts that silicon should be enhanced in regions where the field lines are horizontal (Vauclair et al. 1979, Alecian and Vauclair 1981). In the Doppler images the regions of enhanced silicon usually occur between the presumed location of the magnetic equator and one of the magnetic poles. Often times, as is the case for $56 \mathrm{Ari}$, these enhancements can occur very close to a magnetic pole. If diffusion theory holds then there are two possible explanations for the enhanced silicon regions not occurring exactly at the magnetic equator. Decentering the dipole can displace the band marking the horizontal field lines closer to one of the magnetic poles (Michaud et al. 1981). Alternatively, horizontal diffusion may play an important role in determining the observed distribution of elements of these stars. Mégessier (1984) proposed that silicon may initially accumulate at the magnetic equator, but as the star ages silicon may start to horizontally diffuse toward one or both poles. The close proximity of enhanced silicon near the depleted regions may just be an indication of the evolved status of these stars.

The interpretation of the chromium images is somewhat more complicated. The chromium distributions on $\theta$ Aur and $\epsilon$ UMa are characterized by at least one depleted spot near the magnetic poles as well as a depleted band near the magnetic equator. Such distributions have also been seen on $45 \mathrm{Her}$ and $\omega \mathrm{Her}$, although there are no magnetic field detections that can confirm the relationship of the abundance features with the magnetic field geometries. If chromium diffuses like silicon and is depleted near where the field lines are vertical, then the presence of a depleted band near the magnetic equator in the abundance maps of these stars indicate the presence of a strong quadrupole component. However, the silicon distribution on $\theta$ Aur shows no evidence for a depleted band at the equator and the magnetic field variations can be adequately explained using a pure dipole component (Borra and Landstreet 1980). Also, the chromium distribution on $\gamma^{2}$ Ari is markedly different than that seen on other chromium stars. There are depleted regions of chromium near one magnetic pole but there is no evidence for a depleted band near the expected location of the magnetic equator. A possible solution to this conundrum may again be that horizontal diffusion is playing a prominent role in determining the observed chromium distribution. Chromium may be originally depleted only at the magnetic poles and may be enhanced near the magnetic equator. As the star ages chromium diffuses horizontally to one of the magnetic poles leaving a depleted region (with respect to the mean abundance across the star) at the equator. This may explain why the enhanced chromium regions always appear between the magnetic pole and equator. If this is the case then the differences between the chromium distributions on $\gamma^{2}$ Ari and that of other chromium stars would be attributable to $\gamma^{2}$ Ari being younger than the other chromium stars. 
Table I: Dipole Decentering Parameters

\begin{tabular}{rc}
\hline Star & Decentering $\left(\Delta R_{*}\right)$ \\
\hline$\gamma^{2}$ Ari & 0.20 \\
56 Ari & 0.25 \\
$\theta$ Aur & $\sim 0$ \\
BP Boo & 0.25 \\
$\nu$ For & 0.17 \\
CU Vir & 0.19 \\
45 Her & 0.15 \\
$\omega$ Her & 0.16 \\
$\epsilon$ Uma & 0.03 \\
\hline
\end{tabular}

The silicon distribution on 11 Ori lends some support to the horizontal diffusion hypothesis as the cause of the depleted bands. The radius of this star is anomalously large indicating that it may be an evolved object. Possibly, because of this, the silicon distribution for this object is different than the typical silicon distribution found on Ap stars. In fact, it could be argued that the silicon distribution on 11 Ori looks more like the chromium distribution on several Ap stars (Hatzes 1991ab). Perhaps 11 Ori is old enough where most of the silicon has diffused away from the equator leaving the large depleted structure seen at phase 0.625. It is important to obtain magnetic field measurements of this star so as to determine the exact location of the magnetic pole. Does it coincide with the isolated spot at phase 0.4 (in Fig 5) or does it coincide with the large depleted band-like structure? Interpretations of the silicon image for 11 Ori are premature until these measurements are available.

\section{Inferring the Magnetic Field Geometry from the Abundance Maps}

In most cases the Doppler images exhibit distinguishable features such as spots or bands which can be used to mark the location of the magnetic equator or poles. (At present it seems that the depleted features are more reliable than the enhanced features for marking poles/equators). It may be possible to infer the magnetic field geometries for magnetic Ap stars from the abundance maps if one makes some simplifying assumptions: 1) isolated depleted spots mark the magnetic poles, 2) enhanced silicon regions mark the location of maximum horizontal field strength (as predicted by diffusion theory), 3) the depleted bands in the chromium images mark the magnetic equator, and 4) magnetic fields are produced by decentered dipoles. Admittedly, this is a simplistic approach and the magnetic field geometries so derived are certainly not unique. The presence of stronger higher pole components can modify the distribution of elements without the need for decentering. Also, it is not known what effects, if any, horizontal diffusion plays in modifying the initial distribution of elements. This simple approach is just one means of parameterizing the surface abundance maps.

Fig. 7 shows a histogram of the obliquity angle (angle between magnetic and rotation axis) derived from the Doppler images of the sample of stars listed in Table I. Although the statistical sample is small, there is some evidence for 


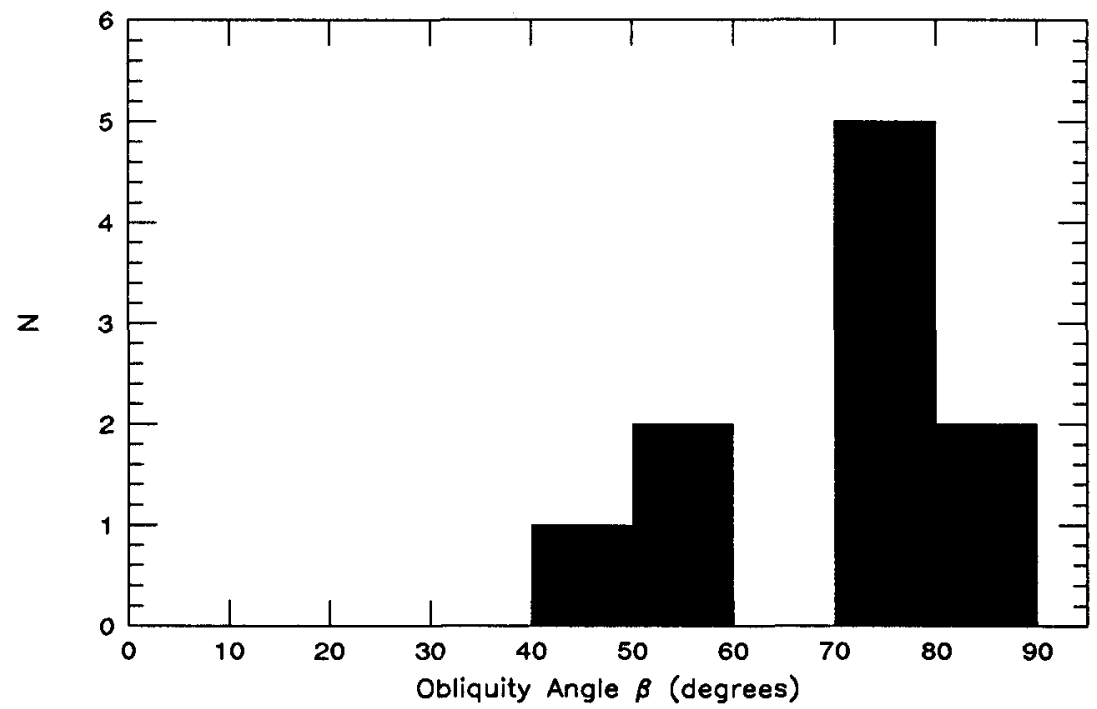

Figure 7: Distribution of obliquity angles $(\beta)$ for the stars in Table I.

bimodality in the distribution with stars having a value of $\beta$ near $50^{\circ}$ or near $75^{\circ}$. None of the sample stars have obliquity angles less than about $40^{\circ}$. However, a larger sample of stars is needed to confirm this result. Preston (1967) found a similar result for slowly rotating Ap stars. Using magnetic field measurements he determined that obliquity angles tended to cluster around $\beta=80^{\circ}$ and $20^{\circ}$. A similar result was also obtained for the rapidly rotating stars by Borra and Landstreet (1980).

Table I lists the decentering parameter, $d$, in fraction of a stellar radius derived from the Doppler images presented here and in previous work. These values represent the magnitude of the decentering parameter and there are many instances (e.g. 56 Ari) where the decentering is not strictly along the dipole axis. Values range from 0.13 to 0.25 of a stellar radius, near the value of 0.15 expected for the size of the convective core in A stars. If the magnetic fields are "fossil" (retained by the star from its formation) then this implies that the convective core destroys magnetic flux and that consequently the dipole is "expelled" from the core. If the field is generated by an internal dynamo then this mechanism must operate just outside the convective core.

However, these decentering parameters may just parameterize the true surface distribution of magnetic flux. Moss (1987ab) has shown that circulation currents in the rapid rotators may redistribute the surface flux. If so, then one may expect a correlation between the amount of decentering and the rotation period and this is indeed seen in Fig. 8 where $d$ is plotted as a function of rotation period for the sample stars. The line represents a least squares fit to the data. The Doppler images may thus show evidence for a redistribution of flux due to rapid rotation as opposed to a true decentering of the dipole. Moss also has demonstrated that for obliquity angles less than about $50^{\circ}$, rapid rotation drives meridional circulation that can submerge the axisymmetric component of the surface field. This should result in no obliquity angles less than about $50^{\circ}$, 


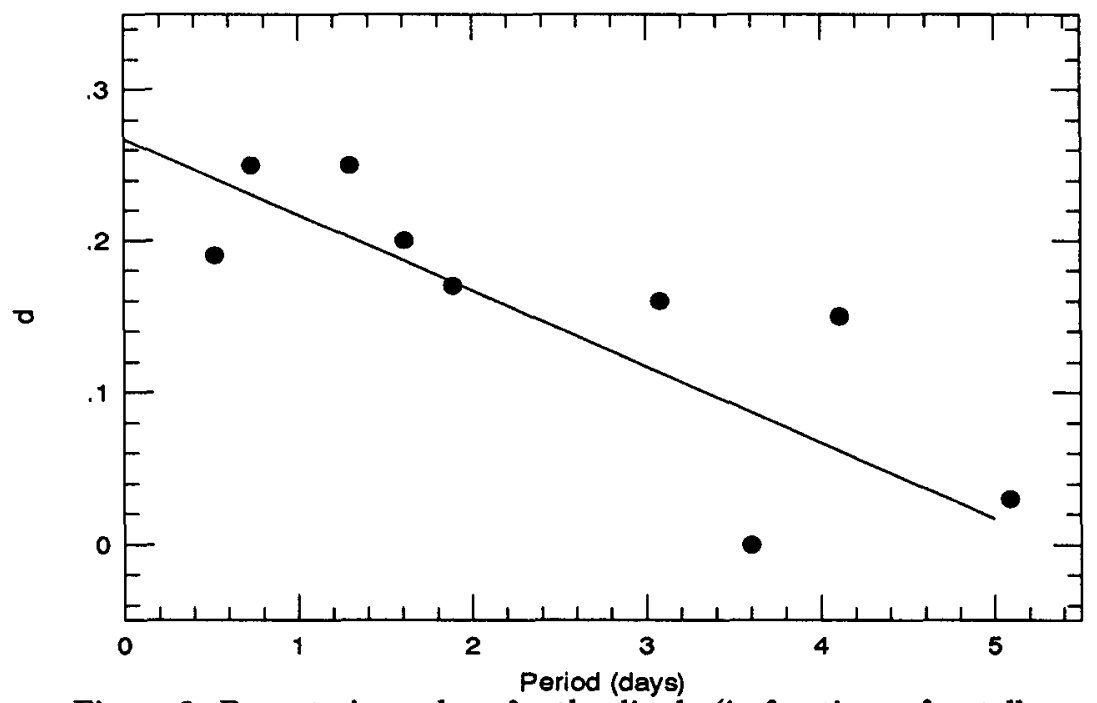

Figure 8: Decentering values for the dipole (in fractions of a stellar radius) as a function of rotation period for the sample of Ap stars

consistent with this study.

\section{Future Work}

Doppler images of abundance features on Ap stars show some promise for use as "magnetograms" for deriving the magnetic field geometries on these stars. This should enable astronomers to determine field geometries in stars where magnetic field measurements are difficult. However, more research is needed before this can be done reliably. Theoretical work is needed to determine the diffusive behavior of elements such as chromium. Is it really depleted where the field lines are vertical and enhanced where the field lines are horizontal? Horizontal diffusion can alter the initial elemental surface distribution so that the Doppler images would imply the presence of higher pole components or decentering of the dipole. Theoretical studies of horizontal diffusion could help determine if this effect is important and how it modifies the initial distribution. This would be difficult as a proper treatment would require numerical studies of diffusion in 3 dimensions with the inclusion of magnetic fields, stellar rotation, and meridional circulation. In observational areas it may be possible to disentangle the effects of horizontal diffusion from dipole decentering by deriving abundance maps for stars of known evolutionary status. Horizontal diffusion would play a more important role in older stars so that systematic differences between the abundance distribution of elements in younger and older stars might be seen. This would require Doppler imaging of Ap stars in clusters of known age. Finally, It is absolutely crucial that magnetic field measurements accompany all Doppler images of Ap stars. These are vital for comparing the observed surface distribution with the location of the magnetic poles and equator. These serve to define a set of rules relating the abundance distribution to the known field geometry that can then be applied to stars where the magnetic fields are too 
weak for detection.

\section{REFERENCES}

Alecian, G. and Vauclair, S., 1981, Astron. Astrophys., 101, 16.

Borra, E.F., and Landstreet, J.D., 1980, Ap. J. Suppl., 42, 421.

Deutsch, A.J. 1955, Pub. A. S. P., 105, 166.

Deutsch, A.J. 1958, Handbk. Phys., 51, 689.

Deutsch, A.J. 1970, Ap. J. , 159, 985

Goncharsky, A.V., Stepanov, V.V., Khokhlova, V.L., and Yagola, A.G. 1982, Astr. Zh., 59, 1146.

Goncharsky, A.V., Ryabchikova, T.A., Stepanov, V.V., Khokhlova, V.L., and Yagola, A.G. 1983, Astr. Zh., 60, 83.

Hatzes, A.P., 1990, Mon. Not. R. astr. Soc., 245, 56.

Hatzes, A.P., 1991a, Mon. Not. R. astr. Soc., 245, 487.

Hatzes, A.P., 1991b, Mon. Not. R. astr. Soc., 253, 487.

Hatzes, A.P., Penrod, G.D., and Vogt, S.S., 1989, Ap. J., 321, 456.

Mégessier, C., 1984, Astron. Astrophys, 138, 267.

Michaud, G., 1970, Ap. J., 160, 640.

Michaud, G., Mégessier, C., and Charland, Y. 1981, Astron. Astrophys., 103, 244.

Moss, D., 1987a Mon. Not. R. astr. Soc., 226, 281.

Moss, D., 1987b Mon. Not. R. astr. Soc., 226, 297.

Preston, G.W., 1967, Ap. J., 150, 547.

Renson, P. and Manfroid, J. 1981, Astron. Astrophys. Suppl. Ser., 23, 413.

Vauclair, S., Hardorp, J., and Peterson, D., 1979, Ap. J., 227, 526.

Vogt, S.S., Penrod, G.D., and Hatzes, A.P., 1987, Ap. J., 321, 496. 